Supporting information for:

\title{
Interfaces Between Different Iron Phthalocyanines and Au(111): Influence of the Fluorination on Structure and Interfacial Interactions
}

Katharina Greulich, ${ }^{\dagger}$ Axel Belser, ${ }^{\dagger}$ Tamara Basova,, Thomas Chassé, ${ }^{+,}{ }^{\ddagger}$ and Heiko Peisert ${ }^{\dagger *}$

${ }^{+}$Institute of Physical and Theoretical Chemistry, University of Tübingen, Auf der Morgenstelle 18, 72076

Tübingen, Germany

" Nikolaev Institute of Inorganic Chemistry SB RAS, 3 Lavrentiev Ave., Novosibirsk, Russia 630090

${ }^{\ddagger}$ Center for Light-Matter Interaction, Sensors \& Analytics (LISA ${ }^{+}$) at the University of Tübingen, Auf der

Morgenstelle 18, 72076 Tübingen, Germany

* Corresponding author, heiko.peisert@uni-tuebingen.de, Tel.: (+49) 07071 / 29-76931, Fax: (+49) 07071 / 295490

\section{Contents}

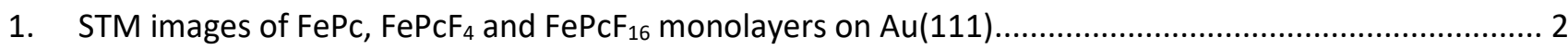

2. $\mathrm{N}$ 1s core level fits of $\mathrm{FePcF}_{\mathrm{x}}$ on $\mathrm{Au}(111)$ as a function of film thickness ............................................. 2

3. $\mathrm{F}$ 1s core levels and $\mathrm{FKLL}$ of $\mathrm{FePcF}_{\mathrm{x}}$ on $\mathrm{Au}(111)$ as a function of film thickness ...................................... 5 


\section{STM images of FePc, $\mathrm{FePcF}_{4}$ and $\mathrm{FePcF}_{16}$ monolayers on $\mathrm{Au(111)}$}

a)

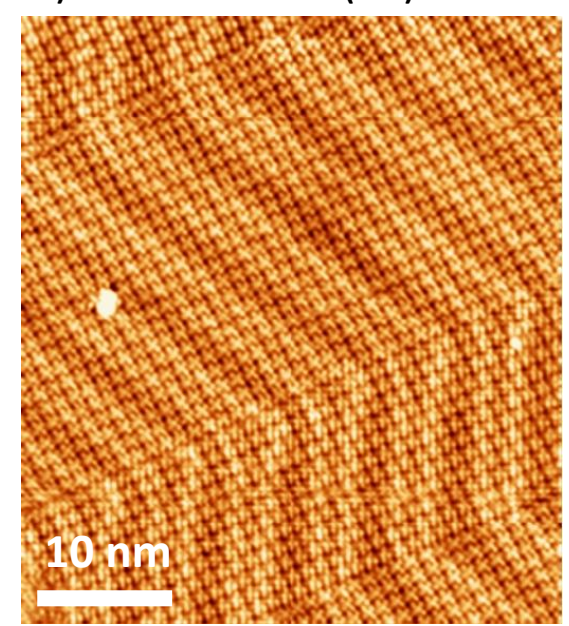

$U_{\text {tip }}=-1.3 \mathrm{VI}=-900 \mathrm{pA}$ b)

$\mathrm{FePcF}_{4}$ on $\mathrm{Au}(111)$

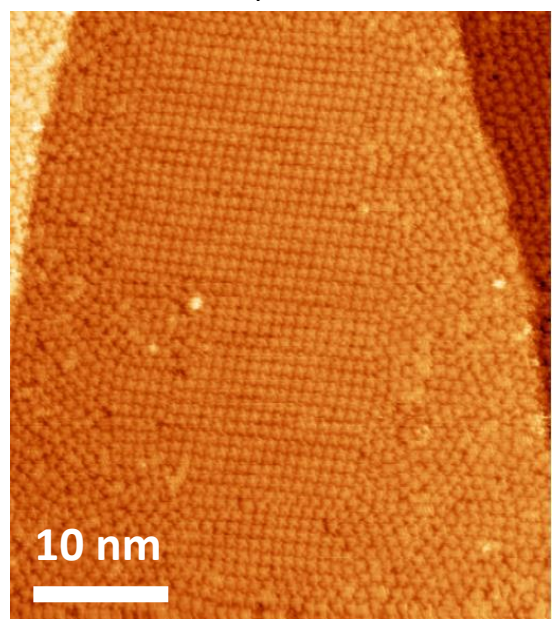

$U_{\text {tip }}=-1.3 \mathrm{VI}=-880 \mathrm{pA}$ c) $\mathrm{FePcF}_{16}$ on $\mathrm{Au}(111)$

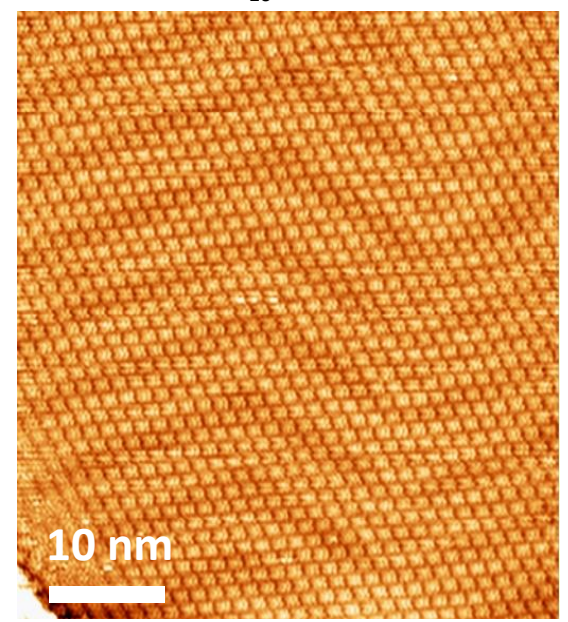

$\mathrm{U}_{\text {tip }}=2.4 \mathrm{VI}=1380 \mathrm{pA}$

Figure S1. Room temperature STM image of a) FePc, b) FePcF 4 and c) $\mathrm{FePcF}_{16}$ monolayers on $\mathrm{Au}(111)$. Compared to the highly order FePc monolayer, the $\mathrm{FePcF}_{4}$ monolayer has unordered regions. In contrast to the other two molecules, only one domain appears in the STM images of FePcF 16 .

2. $\mathbf{N}$ 1s core level fits of $\mathrm{FePcF}_{x}$ on $\mathrm{Au}(\mathbf{1 1 1})$ as a function of film thickness
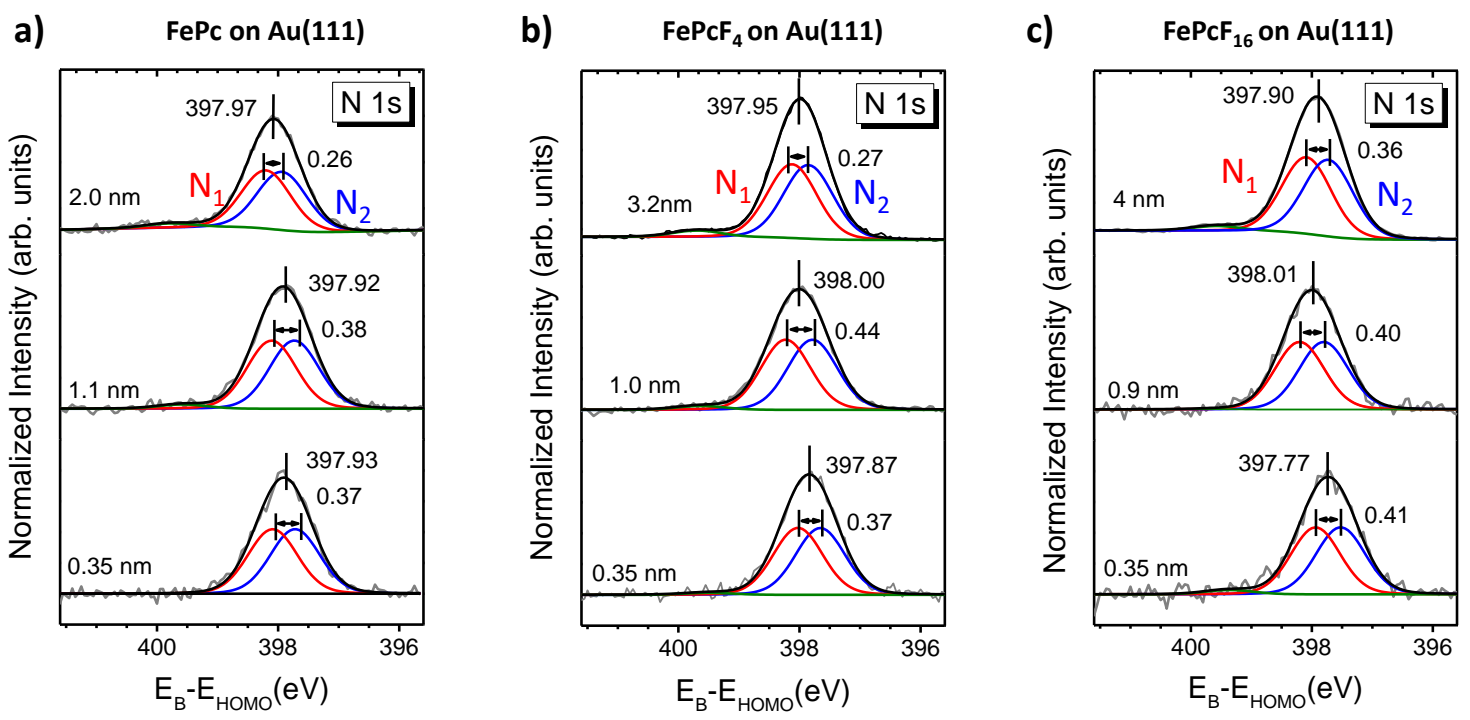

Figure S2. Thickness-dependent $N$ 1s core level fits of a) FePc, b) FePcF $F_{4}$ and c) FePcF $F_{16}$ on $A u(111) . N_{1}$ and $N_{2}$ denote the pyrrolic and the bridging nitrogens in the phthalocyanine rings of the phthalocyanine ring, respectively. All binding energies are references to the energetic position of the HOMO onset. The excitation energy was $h v=1486.7 \mathrm{eV}$.

Table S1. Binding energy positions, Gaussian and Lorentzian widths of the N 1s fit with two components (Fig. S2) of the FePcF $F_{x}$ layers on Au(111). All binding energies are referenced to the HOMO onset ( $\left.E_{B}-E_{\text {Hомо }}\right)$. 


\begin{tabular}{lcccccc}
\hline thickness & $\begin{array}{c}\text { N1 } \\
(\mathrm{nm})\end{array}$ & $\begin{array}{c}\text { N2 } \\
(\mathrm{eV})\end{array}$ & $\begin{array}{c}\text { Sat } \\
(\mathrm{eV})\end{array}$ & $\begin{array}{c}\text { GW } \\
(\mathrm{eV})\end{array}$ & $\begin{array}{c}\text { LW } \\
(\mathrm{eV})\end{array}$ \\
\hline FePc & 0.35 & 398.09 & 397.72 & 399.41 & 0.9 & 0.1 \\
& 0.9 & 398.11 & 397.73 & 399.57 & 0.9 & 0.1 \\
& 2 & 398.20 & 397.94 & 399.78 & 0.9 & 0.1 \\
& & & & & & \\
FePcF $_{4}$ & 0.35 & 398.03 & 397.66 & 399.5 & 0.9 & 0.1 \\
& 1 & 398.23 & 397.79 & 399.63 & 0.9 & 0.1 \\
& 3.2 & 398.12 & 397.85 & 399.69 & 0.9 & 0.1 \\
& & & & & & \\
FePcF $_{16}$ & 0.35 & 397.93 & 397.52 & 399.36 & 0.9 & 0.1 \\
& 0.9 & 398.3 & 397.80 & 399.64 & 0.9 & 0.1 \\
& 4 & 398.10 & 397.73 & 399.57 & 0.9 & 0.1 \\
& & & & & & \\
\hline
\end{tabular}

a)

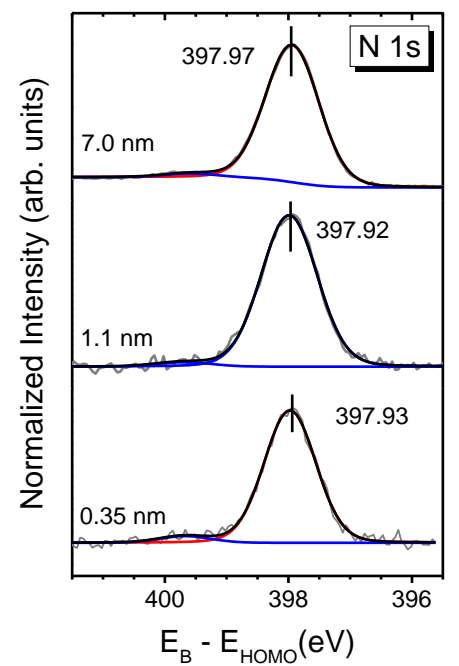

b)

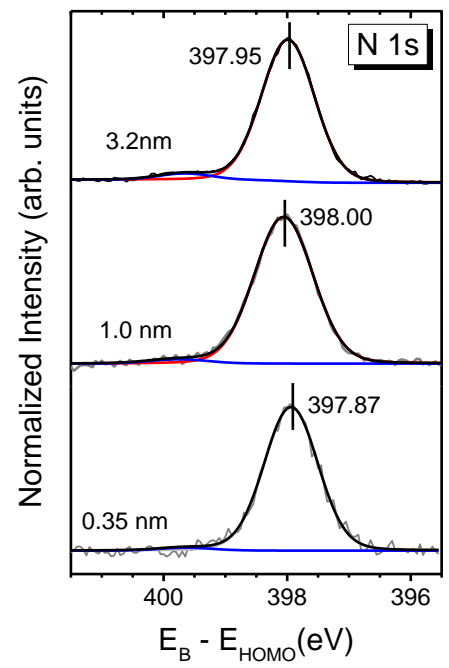

c)

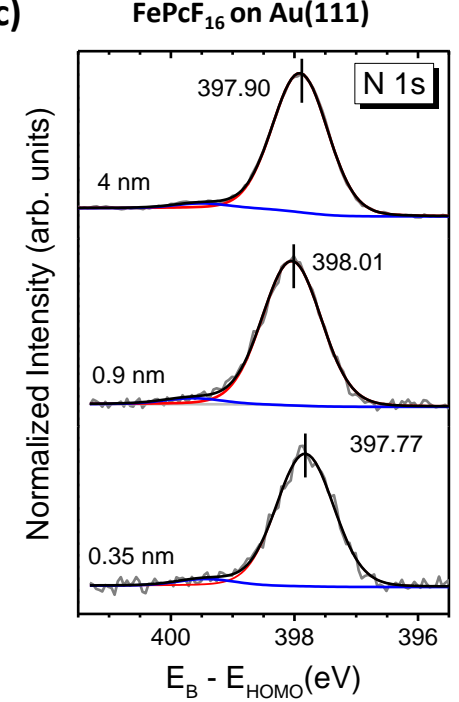

Figure S3. Thickness-dependent $N$ 1s core level fits of a) FePc, b) FePcF4 and c) FePcF16 on Au(111). The stepby-step-deposited monolayer (lowest panel) and the monolayer prepared by multilayer desorption spectra can be fitted with the same fit parameters. All binding energies are references to the energetic position of the HOMO onset. The excitation energy was hv $=1486.7 \mathrm{eV}$.

Table S2. Binding energy positions, Gaussian and Lorentzian widths of the N 1s fit with one component (Fig. S3) of the FePcF layers on $\mathrm{Au}(111)$.

\begin{tabular}{cccccc}
\hline thickness $(\mathrm{nm})$ & $\begin{array}{c}\mathbf{N} \\
(\mathrm{eV})\end{array}$ & $\begin{array}{c}\text { Sat } \\
(\mathrm{eV})\end{array}$ & $\begin{array}{c}\text { GW } \\
(\mathrm{eV})\end{array}$ & $\begin{array}{c}\text { LW } \\
(\mathrm{eV})\end{array}$ \\
\hline FePc & 0.35 & 397.93 & 399.73 & 1.01 & 0.1 \\
& 0.9 & 397.92 & 399.72 & 1.01 & 0.1 \\
& 7 & 397.97 & 399.77 & 0.97 & 0.1
\end{tabular}




\begin{tabular}{cccccc} 
FePcF $_{4}$ & 0.35 & 397.87 & 399.67 & 1.01 & 0.1 \\
& 1 & 398 & 399.8 & 1.12 & 0.1 \\
& 3.2 & 397.95 & 399.75 & 0.96 & 0.1 \\
\multirow{3}{*}{ FePcF $_{16}$} & 0.35 & 397.77 & 399.57 & 1.02 & 0.1 \\
& 0.9 & 398.01 & 399.81 & 1.01 & 0.1 \\
& 4 & 397.9 & 399.7 & 1.01 & 0.1 \\
\hline
\end{tabular}

The $N$ 1s peak of metal phthalocyanines is composed of two individual signals (from the pyrrolic and the bridging nitrogen atoms, components $N_{1}$ and $N_{2}$ represented in red and blue, respectively, in Fig. 5) with an energy separation that is too small to resolve them as individual peaks. For various metal phthalocyanines, energy separations of the $\mathrm{N} 1 \mathrm{~s}$ components between 0.3 and $0.5 \mathrm{eV}$ can be found in the literature. ${ }^{1-5}$ All spectra in Fig. S2 were fitted with components of fixed width (0.9 eV Gaussian, $0.1 \mathrm{eV}$ Lorentzian, fit details are shown in Tab. S1, Supporting Information) and a fixed satellite so that the energy separation of N1 and N2 is the only free parameter. For all three FePcF molecules, this energy separation is slightly larger for the monolayer spectra than for the thin film spectra. The spectra could also be described by assuming a constant energetic separation of $\mathrm{N} 1$ and $\mathrm{N} 2$ peaks and a slight broadening, e. g. caused by molecules adsorbed on not exactly equal adsorption sites, as recently discussed for related phthalocyanines (see Supporting Information, Fig S3 and Tab. S3). ${ }^{6}$ 


\section{F 1s core levels and FKLL of FePcF $F_{x}$ on $A u(111)$ as a function of film thickness}
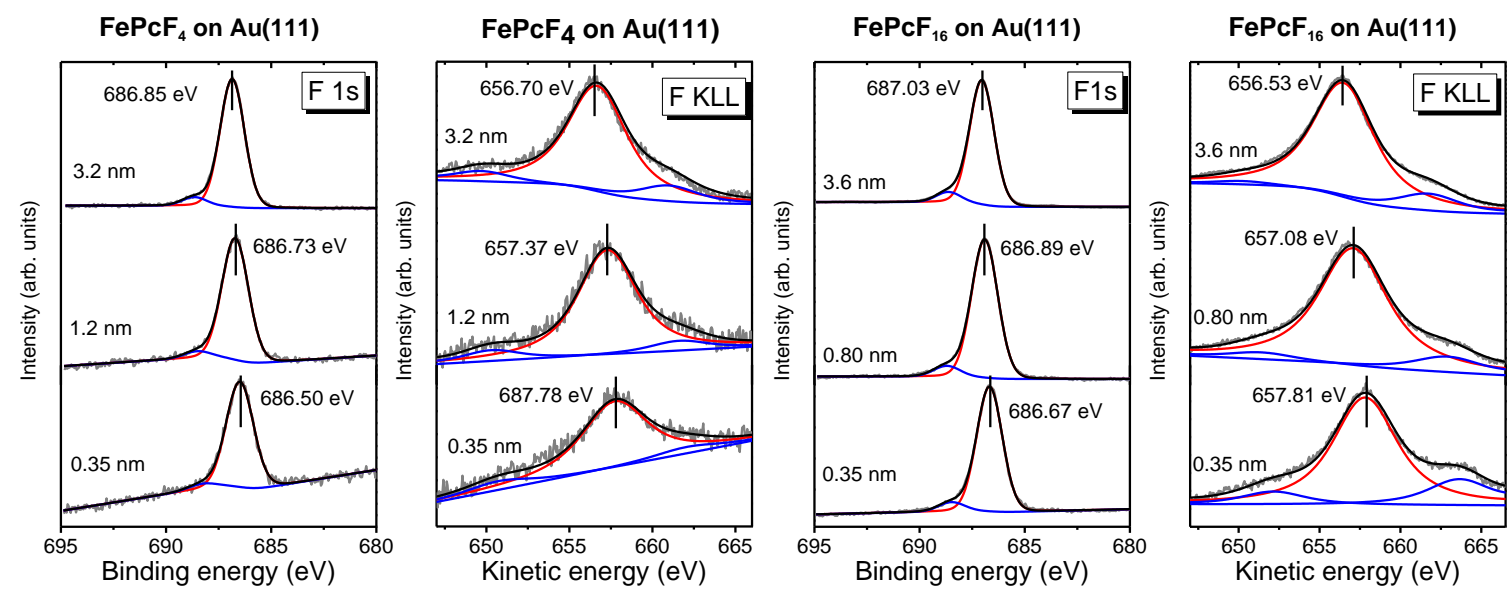

Figure S4. Thickness-dependent F 1s core levels and FKLL of a) FePc, b) FePcF4 and c) FePcF16 on Au(111). All binding energies are references to the energetic position of the HOMO onset. The excitation energy was $h v=1486.7 \mathrm{eV}$.

1. Evangelista, F.; Ruocco, A.; Gotter, R.; Cossaro, A.; Floreano, L.; Morgante, A.; Crispoldi, F.; Betti, M. G.; Mariani, C., Electronic States of CuPc Chains on the Au(110) Surface, J. Chem. Phys. 2009, 131, 174710.

2. Papageorgiou, N.; Ferro, Y.; Salomon, E.; Allouche, A.; Layet, J. M.; Giovanelli, L.; Le Lay, G., Geometry and Electronic Structure of Lead Phthalocyanine: Quantum Calculations Via Density-Functional Theory and Photoemission Measurements, Phys. Rev. B 2003, 68, 235105.

3. Schmid, M.; Kaftan, A.; Steinruck, H. P.; Gottfried, J. M., The Electronic Structure of Cobalt(II) Phthalocyanine Adsorbed on Ag(111), Surf. Sci. 2012, 606, 945-949.

4. Schmid, M.; Zirzlmeier, J.; Steinrück, H.-P.; Gottfried, J. M., Interfacial Interactions of Iron(II) Tetrapyrrole Complexes on Au(111), J. Phys. Chem. C 2011, 115, 17028-17035.

5. Ruocco, A.; Evangelista, F.; Gotter, R.; Attili, A.; Stefani, G., Evidence of Charge Transfer at the CuPhthalocyanine/Ai(100) Lnterface (Reprinted from J. Phys. Chem a, Vol 111, 2007), J. Phys. Chem. C 2008, 112, 2016-2025.

6. Belser, A.; Greulich, K.; Grüninger, P.; Karstens, R.; Ovsyannikov, R.; Giangrisostomi, E.; Nagel, P.; Merz, M.; Schuppler, S.; Chassé, T.; et al., Perfluorinated Phthalocyanines on $\mathrm{Cu}(110)$ and $\mathrm{Cu}(110)-(2 \times 1) \mathrm{O}$ : The Special Role of the Central Cobalt Atom, J. Phys. Chem. C 2021, 125, 8803-8814. 